\title{
Local completeness of locally pseudoconvex spaces and Borwein-Preiss variational principle
}

by

\author{
J. H. QIU (Suzhou) and S. Rolewicz (Warszawa)
}

\begin{abstract}
The notion of local completeness is extended to locally pseudoconvex spaces. Then a general version of the Borwein-Preiss variational principle in locally complete locally pseudoconvex spaces is given, where the perturbation is an infinite sum involving differentiable real-valued functions and subadditive functionals. From this, some particular versions of the Borwein-Preiss variational principle are derived. In particular, a version with respect to the Minkowski gauge of a bounded closed convex set in a locally convex space is presented. In locally convex spaces it can be shown that the relevant perturbation only consists of a single summand if and only if the bounded closed convex set has the quasi-weak drop property if and only if it is weakly compact. From this, a new description of reflexive locally convex spaces is obtained.
\end{abstract}

1. Introduction. Borwein and Preiss gave a smooth variational principle in Banach spaces, which has many applications in nonsmooth analysis and optimization (see [1] and [9]). In terms of $\Phi$-subgradient, Pallaschke and Rolewicz presented a general version of the Borwein-Preiss variational principle in complete metric spaces and gave its interesting applications (see [7, Chapter 2]). In this paper, we first extend the notion of local completeness to locally pseudoconvex spaces. Then we give an extension of the BorweinPreiss variational principle to locally complete locally pseudoconvex spaces, where the perturbation is an infinite sum involving differentiable real-valued functions and subadditive functionals. From the extension, we deduce several versions of the Borwein-Preiss variational principle in various kinds of locally complete locally pseudoconvex spaces. Any closed convex set in a locally convex space yields a subadditive functional, the Minkowski gauge of the set (or of some translation of it). In particular, we give a version of the variational principle with respect to the Minkowski gauge of a bounded

2000 Mathematics Subject Classification: 46A55, 49J45.

Key words and phrases: locally pseudoconvex space, variational principle, quasi-weak drop property, weakly compact set, reflexivity.

Research of J. H. Qiu supported by the National Natural Science Foundation of China (10571035). 
closed convex set in a locally convex space. We consider the problem: under which conditions can the perturbation appearing in the principle consist of a single summand only? In the framework of quasi-complete locally convex spaces, we show that this happens exactly when the bounded closed convex set is weakly compact, or equivalently, has the quasi-weak drop property (on quasi-weak drop property, see $[12,13])$. From this we obtain a characterization of reflexive locally convex spaces by using the Borwein-Preiss variational principle.

2. Locally complete locally pseudoconvex spaces. In this paper, all the spaces $X$ are assumed to be real Hausdorff topological linear spaces (briefly called topological linear spaces). Let $A$ be a nonempty subset of the space $X$. We say that $A$ is starlike if for all $x \in A$ and $0<t \leq 1, t x \in A$. The modulus of concavity (see [15]) of a starlike set $A$ is defined by $c(A)=$ $\inf \{s>0: A+A \subset s A\}$, with the convention that the infimum of an empty set is equal to $+\infty$. Obviously $c(A) \geq 2$ and for a convex set $A, c(A)=2$. A starlike set $A$ with a finite modulus of concavity, $c(A)<\infty$, is called pseudoconvex.

A topological linear space $X$ is called locally pseudoconvex if there is a basis $\left\{U_{\alpha}\right\}$ of neighborhoods of zero consisting of pseudoconvex sets. Let $0<p \leq 1$. We say that a set $A \subset X$ is absolutely $p$-convex if for all $x, y \in A$ and $0 \leq|s|^{p}+|t|^{p} \leq 1, t x+s y \in A$ (see [6]). If $p=1$, then $A$ is called absolutely convex. Obviously, any intersection of absolutely $p$-convex sets is still absolutely $p$-convex. The absolutely $p$-convex hull $\Gamma_{p}(A)$ of a set $A$ is the intersection of all the absolutely $p$-convex sets containing $A$. We observe that $\Gamma_{p}(A)$ consists of all the elements of the form $\sum_{i=1}^{n} t_{i} x_{i}$, where $n \in \mathbb{N}, x_{i} \in A$ for $i=1, \ldots, n$, and $\sum_{i=1}^{n}\left|t_{i}\right|^{p} \leq 1$ (see [16, p. 298]). It is easy to see that if $A$ is absolutely $p$-convex and $0<p^{\prime}<p \leq 1$, then $A$ is automatically absolutely $p^{\prime}$-convex; and hence for any set $A$, $\Gamma_{p^{\prime}}(A) \subset \Gamma_{p}(A)$. For a closed (or open) balanced absorbing set $U$ in $X, U$ is absolutely $p$-convex if and only if \|\|$_{U}$ is a $p$-homogeneous $F$-pseudonorm, where $\|x\|_{U}:=\inf \left\{\lambda>0: x \in \lambda^{1 / p} U\right\}$ for all $x \in X$. It can be shown (see for example [16, pp. 90-93]) that for a locally pseudoconvex space $X$, there is a family $\left\{\|\|_{\alpha}\right\}$ of $p_{\alpha}$-homogeneous $F$-pseudonorms, which determines a topology equivalent to the original one on $X$. Thus the sets of the form $U=\bigcap_{i=1}^{n}\left\{x \in X:\|x\|_{\alpha_{i}} \leq r_{i}\right\}$ constitute a basis of neighborhoods of zero in $X$, where $n \in \mathbb{N},\|\|_{\alpha_{i}}$ is a $p_{\alpha_{i}}$-homogeneous $F$-pseudonorm, $0<p_{\alpha_{i}} \leq 1$ and $r_{i}>0$ for $i=1, \ldots, n$. Since the set $\left\{x \in X:\|x\|_{\alpha_{i}} \leq r_{i}\right\}$ is absolutely $p_{\alpha_{i}}$-convex, it is also absolutely $\min \left\{p_{\alpha_{1}}, \ldots, p_{\alpha_{n}}\right\}$-convex and hence $U=\bigcap_{i=1}^{n}\left\{x \in X:\|x\|_{\alpha_{i}} \leq r_{i}\right\}$ is absolutely $\min \left\{p_{\alpha_{1}}, \ldots, p_{\alpha_{n}}\right\}$-convex. Thus for a topological linear space $X$, the following statements are equivalent: 
(i) $X$ is locally pseudoconvex.

(ii) There is a basis $\left\{U_{\alpha}\right\}$ of neighborhoods of zero consisting of absolutely $p_{\alpha}$-convex sets, where $0<p_{\alpha} \leq 1$.

(iii) There is a family $\left\{\|\|_{\alpha}\right\}$ of $p_{\alpha}$-homogeneous $F$-pseudonorms which determines a topology equivalent to the original one on $X$.

Let the space $X$ be locally pseudoconvex and let $\left\{U_{\alpha}\right\}$ be a basis of neighborhoods of zero which consists of absolutely $p_{\alpha}$-convex sets. If $p:=$ $\inf \left\{p_{\alpha}\right\}>0$, then every $U_{\alpha}$ is absolutely $p$-convex. We say that the space $X$ is locally $p$-convex if there is a basis of neighborhoods of zero consisting of absolutely $p$-convex sets for some fixed $p, 0<p \leq 1$. In particular, if $p=1$, then $X$ is called locally convex. For a locally convex space $X$, we always denote by $X^{*}$ the topological dual of $X$. As is well known, for locally convex spaces there are various kinds of completeness, for example, completeness, quasicompleteness, sequential completeness, $\sum$-completeness, $l^{\infty}$-completeness, local completeness and so on; for details, we refer to [8, pp. 164-165] and [10]. Up to now, local completeness is the weakest known kind of completeness.

Now we extend the notion of local completeness to locally pseudoconvex spaces (concerning local completeness of locally convex spaces, see, for example, $[8$, Chapter 5]) .

Definition 2.1. A subset $B$ of the space $X$ is called a $p$-disc $(0<p \leq 1)$ if it is bounded and absolutely $p$-convex. We denote by $E_{B}$ the linear span of $B, \operatorname{span}[B]$, endowed with the topology induced by the gauge $q_{B}$ of $B$, where $q_{B}(x)=\inf \left\{\lambda>0: x \in \lambda^{1 / p} B\right\}$ for all $x \in \operatorname{span}[B]$. If $E_{B}$ is complete, we call the $p$-disc $B$ a self-complete $p$-disc. A sequence $\left(x_{n}\right)$ in $X$ is called a locally Cauchy sequence if there is a $p \in(0,1]$ and a $p$-disc $B$ in $X$ such that $\left(x_{n}\right)$ is a Cauchy sequence in $E_{B}$. A sequence $\left(x_{n}\right)$ in $X$ is said to be locally convergent to a point $x_{0}$ if there is a $p \in(0,1]$ and a $p$-disc $B$ such that $x_{n} \rightarrow x_{0}$ in $E_{B}$. It is easy to see that $\left(x_{n}\right)$ is locally convergent to $x_{0}$ if and only if $\left(x_{n}-x_{0}\right)$ is locally convergent to zero. A point $x_{0}$ is called a local limit point of a set $A$ in $X$ if there is a sequence $\left(x_{n}\right)$ in $A$ such that $\left(x_{n}\right)$ is locally convergent to $x_{0}$. The set $A$ is called locally closed if every local limit point of $A$ belongs to $A$. Obviously every sequentially closed set is locally closed, but the converse is not true (for an example, see [11, Example 3.1]).

Proposition 2.1. Let $X$ be a locally pseudoconvex space. Then the following statements are equivalent:

(i) For each $p \in(0,1]$ and any $p$-disc $A$, there is a $p^{\prime} \in(0,1]$ and a self-complete $p^{\prime}$-disc $B$ such that $A \subset B$.

(ii) Every locally Cauchy sequence is locally convergent.

(iii) For each $p \in(0,1]$, every closed $p$-disc is a self-complete $p$-disc. 
Proof. (i) $\Rightarrow\left(\right.$ ii). Let $\left(x_{n}\right)$ be a locally Cauchy sequence. Then there is a $p \in(0,1]$ and a $p$-disc $A$ such that $\left(x_{n}\right) \subset \operatorname{span}[A]$ and $\left(x_{n}\right)$ is a Cauchy sequence in $E_{A}$. By (i), there is a $p^{\prime} \in(0,1]$ and a self-complete $p^{\prime}$-disc $B$ such that $A \subset B$. For any $\varepsilon>0$, put $\varepsilon_{1}=\varepsilon^{p / p^{\prime}}$. Then there is $n_{0} \in \mathbb{N}$ such that $x_{n}-x_{m} \in \varepsilon_{1}^{1 / p} A$ for all $m, n \geq n_{0}$. Since $A \subset B$, we have $x_{n}-x_{m} \in$ $\varepsilon_{1}^{1 / p} B=\varepsilon^{1 / p^{\prime}} B$ for all $m, n \geq n_{0}$. That is, $\left(x_{n}\right)$ is a Cauchy sequence in $E_{B}$. Since $E_{B}$ is complete, there exists $x_{0} \in E_{B}$ such that $x_{n} \rightarrow x_{0}$ in $E_{B}$, so $\left(x_{n}\right)$ is locally convergent.

(ii) $\Rightarrow$ (iii). Let $p \in(0,1]$ and $A$ be a closed $p$-disc and let $\left(x_{n}\right)$ be a Cauchy sequence in $E_{A}$. By (ii), $\left(x_{n}\right)$ is locally convergent to some $x_{0}$. Clearly $x_{n} \rightarrow x_{0}$ in $X$. For any $\varepsilon>0$, there is $n_{0} \in \mathbb{N}$ such that $x_{n}-x_{m} \in \varepsilon^{1 / p} A$ for all $m, n \geq n_{0}$. If $m \rightarrow \infty$, then $x_{m} \rightarrow x_{0}$ in $X$. And since $A$ is closed, we have $x_{n}-x_{0} \in \varepsilon^{1 / p} A$ for all $n \geq n_{0}$. This means that $x_{n} \rightarrow x_{0}$ in $E_{A}$ and hence $A$ is a self-complete $p$-disc.

(iii) $\Rightarrow(\mathrm{i})$. Let $A$ be a $p$-disc. Then $\bar{A}$ is also a $p$-disc. By (iii), $\bar{A}$ is a self-complete $p$-disc. By taking $B=\bar{A}$, we complete the proof.

Definition 2.2. A locally pseudoconvex space $X$ is called locally complete if one of the three equivalent statements in Proposition 2.1 is satisfied.

Let $X$ be a locally $p$-convex space $(0<p \leq 1)$. Then a sequence $\left(x_{n}\right)$ in $X$ locally converges to zero if and only if there is an increasing unbounded sequence $\left(\alpha_{n}\right)$ of positive real numbers such that $\left(\alpha_{n} x_{n}\right)$ converges to zero in $X$ (the proof is similar to that of [8, Proposition 5.1.3]). Moreover, $X$ is locally complete if and only if for every bounded set $A$ in $X$ there is a selfcomplete $p$-disc $B$ such that $A \subset B$. We also observe that most statements (except for those involving topological duals) concerning locally complete locally convex spaces can be extended to locally complete locally $p$-convex spaces. Here we do not discuss this in detail.

Next we consider local completeness of locally pseudoconvex spaces (not only locally $p$-convex spaces).

We start with the following obvious

Lemma 2.1. Let $(X,\|\|)$ be an $F^{*}$-space, i.e., a linear space with an F-norm \|\| (see [16, p. 5]). Let $\left(x_{n}\right)$ be a sequence in $X$ convergent to zero. Then there is a sequence $\left(b_{n}\right)$ of positive reals tending to infinity such that $\left(b_{n} x_{n}\right)$ is convergent to zero.

Proof. Without loss of generality we may assume that $x_{n} \neq 0$. We put $b_{n}=E\left(1 / \sqrt{\left\|x_{n}\right\|}\right)$, where $E(t)$ denotes the greatest integer not greater than $t$. Then by the triangle inequality, $\left(b_{n}\right)$ has the required property.

Corollary 2.1. Let $X$ be a topological linear space. Let $\left(x_{n}\right)$ be a sequence in $X$ locally convergent to zero. Then there is a sequence $\left(b_{n}\right)$ of 
positive reals tending to infinity such that $\left(b_{n} x_{n}\right)$ is locally convergent to zero.

Proof. Since $\left(x_{n}\right)$ is locally convergent to 0 , there is a $p \in(0,1]$ and a $p$-disc $B$ such that $\left(x_{n}\right)$ is convergent to 0 with respect to the $p$-homogeneous norm \|\|$_{B}$ induced by $B$. Thus by Lemma 2.1 there is a sequence $\left(b_{n}\right), b_{n}>0$, tending to infinity such that $\left(b_{n} x_{n}\right)$ is convergent to 0 with respect to the $p$-homogeneous norm \|\|$_{B}$. Thus, by definition, $\left(b_{n} x_{n}\right)$ is locally convergent to 0 .

Proposition 2.2. A locally pseudoconvex F-space (i.e. complete metrizable space) $X$ is locally complete.

Proof. Let $\left(x_{n}\right)$ be a locally Cauchy sequence in $X$. Then there is a $p \in(0,1]$ and a closed $p$-disc $B$ such that $\left(x_{n}\right)$ is a Cauchy sequence in $E_{B}=\left(\operatorname{span}[B], q_{B}\right)$. That is, for any $\varepsilon>0$, there is $n_{\varepsilon} \in \mathbb{N}$ such that $x_{n}-x_{m} \in \varepsilon B$ for all $n, m>n_{\varepsilon}$. Since $B$ is bounded in $X,\left(x_{n}\right)$ is also a Cauchy sequence in $X$. And since $X$ is complete, $\left(x_{n}\right)$ is convergent to some $x_{0}$ in $X$. Letting $m \rightarrow \infty$ shows that $x_{n}-x_{0} \in \varepsilon B$ for all $n>n_{\varepsilon}$. This means that $\left(x_{n}\right)$ is convergent to $x_{0}$ in $E_{B}$, hence $\left(x_{n}\right)$ is locally convergent to $x_{0}$ and $X$ is locally complete.

It is of interest to know whether Proposition 2.2 can be reversed. We are able to do it only in locally $p$-convex spaces.

Proposition 2.3. A locally complete locally $p$-convex $F^{*}$-space $(X,\|\|)$ is complete, i.e. it is an F-space.

Proof. Let $\left(x_{n}\right)$ be a Cauchy sequence in $X$ and let $U_{1} \supset U_{2} \supset \cdots$ be a 0 -neighborhood basis consisting of absolutely $p$-convex sets. There is a sequence $n_{1}<n_{2}<\cdots$ such that

$$
x_{n_{i+1}}-x_{n_{i}} \in \frac{1}{2^{i}} U_{i} \quad \text { for all } i \in \mathbb{N} .
$$

Obviously the sequence $\left(2^{i}\left(x_{n_{i+1}}-x_{n_{i}}\right)\right)_{i \in \mathbb{N}}$ is convergent to 0 in $X$. Let $B$ be the closed absolutely $p$-convex hull of the bounded set

$$
\left\{x_{n_{1}}, 2\left(x_{n_{2}}-x_{n_{1}}\right), \ldots, 2^{i}\left(x_{n_{i+1}}-x_{n_{i}}\right), \ldots\right\} .
$$

Then $B$ is a closed $p$-disc, $\left\{x_{n_{1}}, x_{n_{2}}, \ldots\right\} \subset \operatorname{span}[B]$ and $x_{n_{i+1}}-x_{n_{i}} \in 2^{-i} B$. Observe that

$$
\begin{aligned}
x_{n_{i+k}}-x_{n_{i}} & =\left(x_{n_{i+k}}-x_{n_{i+k-1}}\right)+\cdots+\left(x_{n_{i+1}}-x_{n_{i}}\right) \\
& \in \frac{1}{2^{i+k-1}} B+\cdots+\frac{1}{2^{i}} B \subset\left[\left(\frac{1}{2^{i+k-1}}\right)^{p}+\cdots+\left(\frac{1}{2^{i}}\right)^{p}\right]^{1 / p} B \\
& =\frac{1}{2^{i}} \cdot\left(\frac{1-\left(1 / 2^{p}\right)^{k}}{1-1 / 2^{p}}\right)^{1 / p} B \subset \frac{1}{2^{i}} \cdot \frac{2}{\left(2^{p}-1\right)^{1 / p}} B .
\end{aligned}
$$


Since $p>0,\left(x_{n_{i}}\right)_{i \in \mathbb{N}}$ is a Cauchy sequence in $E_{B}=\left(\operatorname{span}[B], q_{B}\right)$. By the hypothesis that $(X,\|\|)$ is locally complete, $E_{B}$ is complete. Hence there is a point $x_{0}$ in $E_{B}$ such that $\left(x_{n_{i}}\right)$ is convergent to $x_{0}$ in $E_{B}$; then clearly $\left(x_{n_{i}}\right)$ is convergent to $x_{0}$ in $(X,\|\|)$. Combining this with the assumption that $\left(x_{n}\right)$ is a Cauchy sequence in $(X,\|\|)$, we conclude that $\left(x_{n}\right)$ is convergent to $x_{0}$ in $(X,\|\|)$, i.e. $(X,\|\|)$ is complete.

Unfortunately this proof does not work in locally pseudoconvex $F^{*}$-spaces as the following example shows.

EXAMPLE 2.1. Let

$$
X=L^{1}[0,1] \times L^{1 / 2}[0,1] \times \cdots \times L^{1 / n}[0,1] \times \cdots
$$

with topology given by pseudonorms

$$
\|x\|_{n}=\left\|\left\{x_{1}(t), \ldots, x_{n}(t), \ldots\right\}\right\|_{n}=\sum_{i=1}^{n} \int_{0}^{1}\left|x_{i}(t)\right|^{1 / i} d t .
$$

Take arbitrary $p, 0<p<1$. In a similar way to the proof that there is no continuous linear functional on $L^{p}[0,1]$ for $p<1$ we can construct a sequence $\left(x^{n}\right)_{n \in \mathbb{N}} \subset X$ converging to 0 such that its absolute $p$-convex hull contains the subspace

$$
X_{n}=\{0\} \times \cdots \times\{0\} \times L^{1 / n}[0,1] \times L^{1 /(n+1)}[0,1] \times \cdots
$$

provided $1 / n<p$. Of course $X_{n}$ is not bounded.

Thus we propose the following open problem to end this section.

Problem 2.1. Is every locally complete locally pseudoconvex $F^{*}$-space $X$ complete?

\section{A version of the Borwein-Preiss principle in locally pseudo-} convex spaces. Let $X$ be a locally pseudoconvex space. For any function $f: X \rightarrow(-\infty, \infty]$, we always assume that $\operatorname{dom} f=\{x \in X: f(x) \neq \infty\}$ is nonempty. A function $f: X \rightarrow(-\infty, \infty]$ is called lower semicontinuous (respectively, sequentially lower semicontinuous) if for any net $\left(x_{n}\right)$ converging to $a$ (respectively, for any sequence $\left(x_{n}\right)$ converging to $a$ ) in $X$, we have $f(a) \leq \liminf _{n} f\left(x_{n}\right)$ (see [5, p. 40] and [18, pp. 149-151]).

As in [14], we call a function $f: X \rightarrow(-\infty, \infty]$ locally lower semicontinuous if for any $r \in \mathbb{R}$, the set $\{x \in X: f(x) \leq r\}$ is locally closed in $X$. This is equivalent to the condition that for any sequence $\left(x_{n}\right)$ locally converging to $a, f(a) \leq \liminf _{n \rightarrow \infty} f\left(x_{n}\right)$. Obviously every (sequentially) lower semicontinuous function is locally lower semicontinuous, and the converse is not true. We denote by $\Phi_{0}$ the class of all strictly increasing, continuous functions $\varphi:[0, \infty) \rightarrow[0, \infty)$ such that $\varphi(0)=0$, and by $\Phi_{1}$ the class of all functions $\varphi:[0, \infty) \rightarrow[0, \infty)$ such that $\varphi(0)=\varphi^{\prime}(0)=0, \varphi^{\prime}(t)$ exists 
and $\varphi^{\prime}(t)>0$ on $(0, \infty)$. Clearly $\Phi_{1} \subset \Phi_{0}$. Moreover $\varphi(\infty)$ always means $\lim _{t \rightarrow \infty} \varphi(t)$ (clearly, it may be $\infty$ ). By modifying the proof of Borwein and Preiss (see [1] or [9, pp. 66-69]), we first give a general version of the Borwein-Preiss variational principle in locally pseudoconvex spaces.

THEOREM 3.1. Let $X$ be a locally complete locally pseudoconvex space, and $p_{0}(\cdot) \leq p_{1}(\cdot) \leq p_{2}(\cdot) \leq \cdots$ be a sequence of locally lower semicontinuous, subadditive, nonnegative functions on $X$ with $p_{i}(0)=0$ such that whenever a sequence $\left(x_{n}\right)$ in $X$ satisfies $p_{i}\left(x_{m}-x_{n}\right) \rightarrow 0(m \geq n \rightarrow \infty)$ for each $i$, then $\left(x_{n}\right)$ is a locally Cauchy sequence in $X$. Let $g: X \rightarrow(-\infty, \infty]$ be a locally lower semicontinuous function, bounded below, and let $x_{0} \in \operatorname{dom} g$, $\varepsilon>g\left(x_{0}\right)-\inf g(X), \lambda>0$ and $\left\{\varphi_{i}: i=0,1,2, \ldots\right\} \subset \Phi_{0}$. Then there exist a sequence $\left(v_{n}\right)$ locally converging to some $v$ in $X$ and $0<\mu<1$ such that

$$
g(x)+\frac{\varepsilon}{\varphi_{0}(\lambda)} \theta(x) \geq g(v)+\frac{\varepsilon}{\varphi_{0}(\lambda)} \theta(v), \quad \forall x \in X,
$$

where $\theta(x)=\sum_{n=0}^{\infty} \mu^{n}(1-\mu) \varphi_{n}\left(p_{n}\left(x-v_{n}\right)\right)$ and $v_{0}=x_{0}$;

(b) $p_{0}\left(v-v_{0}\right) \leq \lambda, p_{0}\left(v_{n}-v_{0}\right) \leq \lambda, p_{n}\left(v-v_{n}\right) \leq \lambda$ for all $n \in \mathbb{N}$, and $p_{n}\left(v-v_{n}\right) \rightarrow 0$

(c) $g(v)<\varepsilon+\inf g(X)$.

Proof. Choose $\varepsilon_{1}>0$ such that

$$
0 \leq g\left(x_{0}\right)-\inf g(X)<\varepsilon_{1}<\varepsilon .
$$

Choose $\mu>0$ such that

$$
0<\mu<1-\varepsilon_{1} / \varepsilon, \quad \text { equivalently, } 0<\varepsilon_{1} / \varepsilon<1-\mu .
$$

Put

$$
\delta=\frac{(1-\mu) \varepsilon}{\varphi_{0}(\lambda)}
$$

From (2) and (3), we know that

$$
\varepsilon_{1} / \delta<\varphi_{0}(\lambda)<\varepsilon / \delta .
$$

For $k=1,2, \ldots$, choose $\gamma_{k}, 0<\gamma_{k}<\mu$, such that

$$
\sum_{k=1}^{\infty} \varphi_{k}^{-1}\left(\frac{\gamma_{1} \cdots \gamma_{k}}{\mu^{k}} \cdot \frac{\varepsilon_{1}}{\delta}\right)<\lambda-\varphi_{0}^{-1}\left(\frac{\varepsilon_{1}}{\delta}\right) .
$$

Put $g_{0}=g$ and $v_{0}=x_{0}$. Define $g_{1}(x)=g_{0}(x)+\delta \varphi_{0}\left(p_{0}\left(x-v_{0}\right)\right), x \in X$. Clearly, $g_{1}\left(x_{0}\right)=g\left(x_{0}\right)<\varepsilon+\inf g(X)<\infty$, hence dom $g_{1} \neq \emptyset$. If $g\left(x_{0}\right)=$ $g_{1}\left(x_{0}\right)=\inf g_{1}(X)$, then we set $v_{1}=v_{0}$. If $g\left(x_{0}\right)=g_{1}\left(x_{0}\right)>\inf g_{1}(X)$, then $\gamma_{1} g\left(x_{0}\right)+\left(1-\gamma_{1}\right) \inf g_{1}(X)>\inf g_{1}(X)$. Hence there exists $v_{1} \in X$ such that $g_{1}\left(v_{1}\right)<\gamma_{1} g\left(x_{0}\right)+\left(1-\gamma_{1}\right) \inf g_{1}(X)$. Thus in any case, there exists $v_{1} \in X$ such that

$$
g_{1}\left(v_{1}\right) \leq \gamma_{1} g\left(x_{0}\right)+\left(1-\gamma_{1}\right) \inf g_{1}(X) .
$$


In general, define

$$
g_{n+1}(x)=g_{n}(x)+\delta \mu^{n} \varphi_{n}\left(p_{n}\left(x-v_{n}\right)\right), \quad x \in X .
$$

Clearly $g_{n+1}\left(v_{n}\right)=g_{n}\left(v_{n}\right)<\infty$, hence dom $g_{n+1} \neq \emptyset$. Choose $v_{n+1} \in X$ such that

$$
g_{n+1}\left(v_{n+1}\right) \leq \gamma_{n+1} g_{n}\left(v_{n}\right)+\left(1-\gamma_{n+1}\right) \inf g_{n+1}(X) .
$$

Put $s_{n}=\inf g_{n}(X)$ and $a_{n}=g_{n}\left(v_{n}\right)$. Then

$$
\begin{aligned}
a_{n+1}=g_{n+1}\left(v_{n+1}\right) & \leq \gamma_{n+1} g_{n}\left(v_{n}\right)+\left(1-\gamma_{n+1}\right) \inf g_{n+1}(X) \\
& \leq \gamma_{n+1} g_{n}\left(v_{n}\right)+\left(1-\gamma_{n+1}\right) g_{n+1}\left(v_{n}\right) \\
& =\gamma_{n+1} g_{n}\left(v_{n}\right)+\left(1-\gamma_{n+1}\right) g_{n}\left(v_{n}\right) \\
& =g_{n}\left(v_{n}\right)=a_{n} .
\end{aligned}
$$

Obviously $g_{n} \leq g_{n+1}$, hence

$$
\begin{aligned}
s_{n} \leq s_{n+1} & \leq a_{n+1}=g_{n+1}\left(v_{n+1}\right) \leq \gamma_{n+1} g_{n}\left(v_{n}\right)+\left(1-\gamma_{n+1}\right) \inf g_{n+1}(X) \\
& =\gamma_{n+1} a_{n}+\left(1-\gamma_{n+1}\right) s_{n+1} .
\end{aligned}
$$

From this, we have

$$
\begin{aligned}
0 \leq a_{n+1}-s_{n+1} & \leq \gamma_{n+1}\left(a_{n}-s_{n+1}\right) \leq \gamma_{n+1}\left(a_{n}-s_{n}\right) \leq \cdots \\
& \leq \gamma_{n+1} \gamma_{n} \cdots \gamma_{1}\left(a_{0}-s_{0}\right)<\gamma_{n+1} \gamma_{n} \cdots \gamma_{1} \varepsilon_{1}
\end{aligned}
$$

Taking $x=v_{n+1}$ in (5), we have

$$
\begin{aligned}
a_{n+1}=g_{n+1}\left(v_{n+1}\right) & =g_{n}\left(v_{n+1}\right)+\delta \mu^{n} \varphi_{n}\left(p_{n}\left(v_{n+1}-v_{n}\right)\right) \\
& \geq s_{n}+\delta \mu^{n} \varphi_{n}\left(p_{n}\left(v_{n+1}-v_{n}\right)\right) .
\end{aligned}
$$

From (7)-(9), we obtain

$$
\delta \mu^{n} \varphi_{n}\left(p_{n}\left(v_{n+1}-v_{n}\right)\right) \leq a_{n+1}-s_{n} \leq a_{n}-s_{n}<\gamma_{n} \gamma_{n-1} \cdots \gamma_{1} \varepsilon_{1} .
$$

Therefore,

$$
p_{n}\left(v_{n+1}-v_{n}\right) \leq \varphi_{n}^{-1}\left(\frac{\gamma_{1} \cdots \gamma_{n}}{\mu^{n}} \cdot \frac{\varepsilon_{1}}{\delta}\right) \text {. }
$$

For each $i \in \mathbb{N}, n \geq i$ and $j \in \mathbb{N}$, we have

$$
\begin{aligned}
p_{i}\left(v_{n+j}-v_{n}\right) & \leq p_{n}\left(v_{n+j}-v_{n}\right) \\
& \leq p_{n}\left(v_{n+j}-v_{n+j-1}\right)+\cdots+p_{n}\left(v_{n+1}-v_{n}\right) \\
& \leq p_{n+j-1}\left(v_{n+j}-v_{n+j-1}\right)+\cdots+p_{n}\left(v_{n+1}-v_{n}\right) \\
& <\sum_{k=n}^{\infty} \varphi_{k}^{-1}\left(\frac{\gamma_{1} \cdots \gamma_{k}}{\mu^{k}} \cdot \frac{\varepsilon_{1}}{\delta}\right) .
\end{aligned}
$$

Combining this with (4), we conclude that for each $i \in \mathbb{N}, p_{i}\left(v_{m}-v_{n}\right) \rightarrow 0$ when $m \geq n \rightarrow \infty$. This implies that $\left(v_{n}\right)$ is a locally Cauchy sequence in $X$. Since $X$ is locally complete, there exists $v \in X$ such that $\left(v_{n}\right)$ is locally 
convergent to $v$. Letting $j \rightarrow \infty$ on the left of $(10)$ shows that $\left(v_{n+j}\right)_{j}$ is locally convergent to $v$; and since every $p_{i}$ is locally lower semicontinuous, we have

$$
p_{n}\left(v-v_{n}\right) \leq \sum_{k=n}^{\infty} \varphi_{k}^{-1}\left(\frac{\gamma_{1} \cdots \gamma_{k}}{\mu^{k}} \cdot \frac{\varepsilon_{1}}{\delta}\right)<\lambda-\varphi_{0}^{-1}\left(\frac{\varepsilon_{1}}{\delta}\right), \quad \forall n \in \mathbb{N} .
$$

From (11), we obtain

$$
p_{n}\left(v-v_{n}\right)<\lambda \quad \text { and } \quad p_{n}\left(v-v_{n}\right) \rightarrow 0 \quad(n \rightarrow \infty) .
$$

Since

$$
\begin{aligned}
\delta \varphi_{0}\left(p_{0}\left(v_{1}-v_{0}\right)\right) & =g_{1}\left(v_{1}\right)-g_{0}\left(v_{1}\right) \\
& \leq g_{1}\left(v_{1}\right)-\inf g(X)=a_{1}-s_{0} \leq a_{0}-s_{0}<\varepsilon_{1},
\end{aligned}
$$

we have

$$
p_{0}\left(v_{1}-v_{0}\right)<\varphi_{0}^{-1}\left(\varepsilon_{1} / \delta\right) .
$$

From (11) and (13), we deduce that

$$
\begin{aligned}
p_{0}\left(v-v_{0}\right) & \leq p_{0}\left(v-v_{1}\right)+p_{0}\left(v_{1}-v_{0}\right) \leq p_{1}\left(v-v_{1}\right)+p_{0}\left(v_{1}-v_{0}\right) \\
& <\lambda-\varphi_{0}^{-1}\left(\varepsilon_{1} / \delta\right)+\varphi_{0}^{-1}\left(\varepsilon_{1} / \delta\right)=\lambda .
\end{aligned}
$$

Taking $n=i=1$ in (10), for any $j \in \mathbb{N}$ we get

$$
p_{1}\left(v_{1+j}-v_{1}\right)<\sum_{k=1}^{\infty} \varphi_{k}^{-1}\left(\frac{\gamma_{1} \cdots \gamma_{k}}{\mu^{k}} \cdot \frac{\varepsilon_{1}}{\delta}\right)<\lambda-\varphi_{0}^{-1}\left(\frac{\varepsilon_{1}}{\delta}\right) .
$$

From this, we have

$$
\begin{aligned}
p_{0}\left(v_{1+j}-v_{0}\right) & \leq p_{0}\left(v_{1+j}-v_{1}\right)+p_{0}\left(v_{1}-v_{0}\right) \\
& \leq p_{1}\left(v_{1+j}-v_{1}\right)+p_{0}\left(v_{1}-v_{0}\right) \\
& <\lambda-\varphi_{0}^{-1}\left(\varepsilon_{1} / \delta\right)+\varphi_{0}^{-1}\left(\varepsilon_{1} / \delta\right)=\lambda .
\end{aligned}
$$

By (13)-(15), we conclude that

$$
p_{0}\left(v_{n}-v_{0}\right) \leq \lambda \text { and } p_{0}\left(v-v_{0}\right) \leq \lambda .
$$

Combining this with (12), we see that (b) holds.

From (7), $a_{0} \geq a_{1} \geq a_{2} \geq \cdots$. Since $a_{n}=g_{n}\left(v_{n}\right) \geq \inf g_{n}(X)>-\infty$, we infer that $\lim _{n \rightarrow \infty} a_{n}$ exists and is finite. By (8),

$$
0 \leq a_{n}-s_{n}<\gamma_{1} \cdots \gamma_{n} \varepsilon_{1}<\mu^{n} \varepsilon_{1} \rightarrow 0 \quad(n \rightarrow \infty),
$$

hence $\lim _{n \rightarrow \infty} s_{n}=\lim _{n \rightarrow \infty} a_{n}$ exists and is finite. Put

$$
\theta(x)=\sum_{n=0}^{\infty} \mu^{n}(1-\mu) \varphi_{n}\left(p_{n}\left(x-v_{n}\right)\right) .
$$


Then for any $x \in \operatorname{dom} g$, we have

$$
\begin{aligned}
g(x)+\frac{\varepsilon}{\varphi_{0}(\lambda)} \theta(x) & =g(x)+\frac{\varepsilon(1-\mu)}{\varphi_{0}(\lambda)} \sum_{n=0}^{\infty} \mu^{n} \varphi_{n}\left(p_{n}\left(x-v_{n}\right)\right) \\
& =g(x)+\delta \sum_{n=0}^{\infty} \mu^{n} \varphi_{n}\left(p_{n}\left(x-v_{n}\right)\right) \\
& =\sup _{n} g_{n}(x) \geq \lim _{n \rightarrow \infty} s_{n}=\lim _{n \rightarrow \infty} a_{n}=\lim _{n \rightarrow \infty} g_{n}\left(v_{n}\right) .
\end{aligned}
$$

Let $\alpha=\sup _{m} \liminf g_{m}\left(v_{n}\right)$. Then for any $\eta>0$, there exists $m \in \mathbb{N}$ such that $\liminf _{n} g_{m}\left(v_{n}\right)>\alpha-\eta$. Hence there are $n_{1}<n_{2}<\cdots$ such that $g_{m}\left(v_{n_{i}}\right)>\alpha-\eta$. Take $i$ large enough so that $n_{i} \geq m$. Then

$$
g_{n_{i}}\left(v_{n_{i}}\right) \geq g_{m}\left(v_{n_{i}}\right)>\alpha-\eta .
$$

Also, $\lim _{n \rightarrow \infty} a_{n}=\lim _{n \rightarrow \infty} g_{n}\left(v_{n}\right)$ exists, so we have $\lim _{n \rightarrow \infty} g_{n}\left(v_{n}\right) \geq \alpha$. From this and (16), we obtain

$$
g(x)+\frac{\varepsilon}{\varphi_{0}(\lambda)} \theta(x) \geq \alpha=\sup _{m} \liminf _{n} g_{m}\left(v_{n}\right) .
$$

Since $g_{m}$ is locally lower semicontinuous and $\left(v_{n}\right)$ is locally convergent to $v$, it follows that $\liminf g_{n} g_{m}\left(v_{n}\right) \geq g_{m}(v)$. Combining this with (17), we conclude that

$$
g(x)+\frac{\varepsilon}{\varphi_{0}(\lambda)} \theta(x) \geq \sup _{m} g_{m}(v)=g(v)+\frac{\varepsilon}{\varphi_{0}(\lambda)} \theta(v),
$$

i.e. (a) holds.

Finally, we show that (c) holds. Since $g_{0}\left(v_{0}\right)=g\left(x_{0}\right)<\varepsilon_{1}+\inf g(X)$,

$$
\begin{aligned}
g_{1}\left(v_{1}\right) & \leq \gamma_{1} g\left(x_{0}\right)+\left(1-\gamma_{1}\right) \inf g_{1}(X) \\
& \leq \gamma_{1} g\left(x_{0}\right)+\left(1-\gamma_{1}\right) g_{1}\left(x_{0}\right)=g\left(x_{0}\right)<\varepsilon_{1}+\inf g(X) .
\end{aligned}
$$

By $(7),\left(a_{n}\right)$ is decreasing, hence for any $n \in \mathbb{N}$,

$$
a_{n}=g_{n}\left(v_{n}\right) \leq a_{1}<\varepsilon_{1}+\inf g(X) .
$$

Therefore

$$
\begin{aligned}
g(v) & \leq g(v)+\frac{\varepsilon}{\varphi_{0}(\lambda)} \theta(v)=\sup _{m} g_{m}(v) \\
& \leq \sup _{m} \liminf _{n} g_{m}\left(v_{n}\right)=\alpha \leq \lim _{n \rightarrow \infty} g_{n}\left(v_{n}\right)<\varepsilon_{1}+\inf g(X) .
\end{aligned}
$$

As we have seen in Section 2, in a metrizable locally $p$-convex space $(0<p \leq 1)$, a sequence is convergent if and only if it is locally convergent. Hence a function defined on the space is (sequentially) lower semicontinuous if and only if it is locally lower semicontinuous. Moreover by Proposition 2.2, a complete metrizable locally pseudoconvex space is locally complete. Now let $X$ be a complete metrizable locally pseudoconvex space and \|\|$_{0} \leq\|\|_{1} \leq$ \|\|$_{2} \leq \cdots$ be a sequence of $p_{n}$-homogeneous $F$-pseudonorms determining the 
topology of $X$ (see [16, pp. 90-95]). By substituting the sequence \|\|$_{0} \leq$ \|\|$_{1} \leq\|\|_{2} \leq \cdots$ for the sequence $p_{0} \leq p_{1} \leq p_{2} \leq \cdots$ in Theorem 3.1, we immediately obtain the following result, which may be useful in studying the differentiability of perturbations.

Corollary 3.1. Let $X$ be a complete metrizable locally pseudoconvex space with the topology generated by an increasing sequence \|\|$_{0} \leq\|\|_{1} \leq$ \|\|$_{2} \leq \cdots$ of $p_{n}$-homogeneous $F$-pseudonorms. Let $g: X \rightarrow(-\infty, \infty]$ be a lower semicontinuous function bounded below, let $x_{0}, \varepsilon>0$, and $\lambda>0$ be as in Theorem 3.1, and let $\left\{\varphi_{n}: n=0,1,2, \ldots\right\} \subset \Phi_{0}$. Then there exist a sequence $\left(v_{n}\right)$ converging to $v$ in $X$ and $0<\mu<1$ such that

$$
g(x)+\frac{\varepsilon}{\varphi_{0}(\lambda)} \theta(x) \geq g(v)+\frac{\varepsilon}{\varphi_{0}(\lambda)} \theta(v), \quad \forall x \in X,
$$

where $\theta(x)=\sum_{n=0}^{\infty} \mu^{n}(1-\mu) \varphi_{n}\left(\left\|x-v_{n}\right\|_{n}\right)$ and $v_{0}=x_{0}$;

(b) $\left\|v-v_{0}\right\|_{0} \leq \lambda,\left\|v_{n}-v_{0}\right\|_{0} \leq \lambda,\left\|v-v_{n}\right\|_{n} \leq \lambda$ for all $n \in \mathbb{N}$, and $\left\|v-v_{n}\right\|_{n} \rightarrow 0$

(c) $g(v)<\varepsilon+\inf g(X)$.

Let $B \subset X$ be a $p$-convex set (i.e. $s B+t B \subset B$ for all $s, t \geq 0$ such that $\left.s^{p}+t^{p}=1\right)$ with $0 \in B(0<p \leq 1)$. Then $B$ induces in $X$ a Minkowski gauge $q_{B}: X \rightarrow[0, \infty]$ as follows: $q_{B}(x)= \begin{cases}\inf \left\{\lambda>0: x \in \lambda^{1 / p} B\right\} & \text { if there exists } \lambda>0 \text { such that } x \in \lambda^{1 / p} B \\ \infty & \text { else. }\end{cases}$

The Minkowski gauge $q_{B}$ is positive $p$-homogeneous and subadditive.

Corollary 3.2. Let $X$ be a locally complete locally p-convex space $(0<p \leq 1)$, and $B \subset X$ be a locally closed bounded p-convex set with $0 \in B$. Let $g: X \rightarrow(-\infty, \infty]$ be a locally lower semicontinuous function bounded below and let $x_{0} \in \operatorname{dom} g, \varepsilon>g\left(x_{0}\right)-\inf g(X), \lambda>0$ and $\varphi \in \Phi_{0}$. Then there exist a sequence $\left(v_{n}\right)$ locally converging to $v$ in $X$ and $0<\mu<1$ such that

$$
g(x)+\frac{\varepsilon}{\varphi(\lambda)} \theta(x) \geq g(v)+\frac{\varepsilon}{\varphi(\lambda)} \theta(v), \quad \forall x \in X,
$$

where $\theta(x)=\sum_{n=0}^{\infty} \mu^{n}(1-\mu) \varphi\left(q_{B}\left(x-v_{n}\right)\right)$ and $v_{0}=x_{0}$;

(b) $q_{B}\left(v-v_{0}\right) \leq \lambda, q_{B}\left(v_{n}-v_{0}\right) \leq \lambda, q_{B}\left(v-v_{n}\right) \leq \lambda$ for all $n \in \mathbb{N}$, and $q_{B}\left(v-v_{n}\right) \rightarrow 0$

(c) $g(v)<\varepsilon+\inf g(X)$.

Proof. Clearly, the Minkowski gauge $q_{B}$ of the locally closed bounded $p$-convex set $B$ is locally lower semicontinuous and subadditive. Denote the absolutely $p$-convex hull of $B$ by $D$; it is a $p$-disc. Thus if a sequence $\left(x_{n}\right)$ 
in $X$ satisfies $q_{B}\left(x_{m}-x_{n}\right) \rightarrow 0(m \geq n \rightarrow \infty)$, then $q_{D}\left(x_{m}-x_{n}\right) \rightarrow 0$ $(m \geq n \rightarrow \infty)$. That is to say, $\left(x_{n}\right)$ is a locally Cauchy sequence in $X$. Now applying Theorem 3.1, we immediately obtain the result.

As a special case of Corollary 3.2, we have the following:

Corollary 3.3. Let $X$ be a quasi-complete (or sequentially complete, or locally complete) locally convex space and $B \subset X$ a bounded closed convex set with $0 \in B$. Let $g: X \rightarrow(-\infty, \infty]$ be a lower semicontinuous function bounded below, and let $x_{0} \in \operatorname{dom} g, \varepsilon>g\left(x_{0}\right)-\inf g(X), \lambda>0$ and $\varphi \in \Phi_{0}$. Then there exist a sequence $\left(v_{n}\right)$ converging to $v$ in $X$ and $0<\mu<1$ such that (a)-(c) in Corollary 3.2 hold.

In fact, in view of [7] we see that in Theorem 3.1 and Corollaries 3.1-3.3, the function

$$
-\frac{\varepsilon}{\varphi_{0}(\lambda)} \theta(x) \quad \text { or } \quad-\frac{\varepsilon}{\varphi(\lambda)} \theta(x)
$$

is a $\Phi$-subgradient of $g$ at the point $v$, where $\Phi$ denotes the appropriate class of functions according to different cases.

\section{Perturbation only consisting of a single summand and reflex-} ivity of locally convex spaces. As mentioned in [9, p. 71], even in the framework of Banach spaces, it is not always possible to assume that the perturbation function $\theta$ used in the variational principle consists of a single summand. Borwein and Preiss [1] have shown that this is possible in reflexive Banach spaces and that these are the only spaces with this property. In this section, we consider the following problem: under which condition, can $\theta$ appearing in Corollary 3.3 consist of a single summand only? This problem will be solved in the framework of quasi-complete locally convex spaces. We shall see that this happens exactly when $B$ is weakly compact. From this we obtain a criterion for reflexivity of locally convex spaces. In this section we denote by $\Phi_{2}$ the class of all functions $\varphi:[0, \infty) \rightarrow[0, \infty)$ such that $\varphi(0)=\varphi^{\prime}(0)=0, \varphi^{\prime}(t)$ exists and strictly increases on $[0, \infty)$. Clearly $\Phi_{2} \subset \Phi_{1} \subset \Phi_{0}$.

THEOREM 4.1. Let $X$ be a locally convex space and $B \subset X$ be a bounded closed convex set with $0 \in B$. If for any lower semicontinuous convex function $g: X \rightarrow(-\infty, \infty]$ bounded below, any $x_{0} \in \operatorname{dom} g$, any $\varepsilon>g\left(x_{0}\right)-$ $\inf g(X)$ and any $\lambda>0$, there exist $\varphi \in \Phi_{2}$ and $v \in X$ such that

$$
g(x)+\frac{\varepsilon}{\varphi(\lambda)} \varphi\left(q_{B}\left(x-x_{0}\right)\right) \geq g(v)+\frac{\varepsilon}{\varphi(\lambda)} \varphi\left(q_{B}\left(v-x_{0}\right)\right) \quad \text { for all } x \in X,
$$

then for any $x^{*} \in X^{*}$, there exists $u \in B$ such that $\left\langle x^{*}, u\right\rangle=\sup \left\langle x^{*}, B\right\rangle$.

Proof. For any fixed $x^{*} \in X^{*}$, if $\left\langle x^{*}, B\right\rangle \leq 0$, we may take $u=0$. If not, there exists $b \in B$ such that $\left\langle x^{*}, b\right\rangle>0$. Since $\left\langle x^{*}, B\right\rangle$ is bounded, there exists 
$\alpha>0$ such that $\left|\left\langle x^{*}, B\right\rangle\right| \leq \alpha$. Without loss of generality, we may assume that $\alpha<1$, or else we may consider $k x^{*}$, where $k>0$ is small enough. Put $U:=\left\{x \in X:\left|\left\langle x^{*}, x\right\rangle\right| \leq 1\right\}$. Then $U$ is a 0 -neighborhood in $X$ and $B \subset U^{\mathrm{i}}$, where $U^{\mathrm{i}}$ denotes the interior of $U$. Define

$$
g(x)= \begin{cases}-\left\langle x^{*}, x\right\rangle & \text { if } x \in U \\ \infty & \text { if } x \notin U\end{cases}
$$

Clearly, $g: X \rightarrow(-\infty, \infty]$ is a lower semicontinuous convex function, bounded below. Set $x_{0}=0, \varepsilon=1$. Then

$$
\varepsilon=1>\alpha \geq g\left(x_{0}\right)-\inf g(X) .
$$

By hypothesis, for $\lambda=1$, there exist $\varphi \in \Phi_{2}$ and $v \in X$ such that $q_{B}\left(v-x_{0}\right)=$ $q_{B}(v) \leq 1$ and

$$
g(x)+\frac{1}{\varphi(1)} \varphi\left(q_{B}(x)\right) \geq g(v)+\frac{1}{\varphi(1)} \varphi\left(q_{B}(v)\right), \quad x \in X .
$$

It is also clear that $x \mapsto \frac{1}{\varphi(1)} \varphi\left(q_{B}(x)\right)$ is a lower semicontinuous convex function, bounded below, and $g$ is continuous at $x=0$. By (18) and the MoreauRockafeller theorem (for example, see [18, Theorem 47.B, pp. 389-390]),

$$
\left.\left.0 \in \partial\left(g+\frac{1}{\varphi(1)} \varphi\left(q_{B}(\cdot)\right)\right)\right|_{v} \subset \partial g\right|_{v}+\left.\frac{1}{\varphi(1)} \partial \varphi\left(q_{B}(\cdot)\right)\right|_{v} .
$$

Let $\left.z^{*} \in \partial g\right|_{v}$. Then for any $x \in X$,

$$
\left\langle z^{*}, x\right\rangle-\left\langle z^{*}, v\right\rangle \leq g(x)-g(v) .
$$

In particular, when $x \in U$, we have

$$
\left\langle z^{*}, x\right\rangle-\left\langle z^{*}, v\right\rangle \leq-\left\langle x^{*}, x\right\rangle+\left\langle x^{*}, v\right\rangle .
$$

That is,

$$
\left\langle z^{*}+x^{*}, x-v\right\rangle \leq 0, \quad \forall x \in U .
$$

Since $q_{B}(v) \leq 1$, we have $v \in B$, which implies that $v \in U^{\mathrm{i}}$. Thus $U-v$ is a 0 -neighborhood in $X$. Since the 0-neighborhood $U-v$ contains a balanced 0 -neighborhood, combining this and (20), we conclude that $z^{*}+x^{*}=0$, i.e. $z^{*}=-x^{*}$. Hence $\left.\partial g\right|_{v}=\left\{-x^{*}\right\}$. Thus (19) becomes

$$
0 \in\left\{-x^{*}\right\}+\left.\frac{1}{\varphi(1)} \partial \varphi\left(q_{B}(\cdot)\right)\right|_{v}
$$

From this,

$$
\left.x^{*} \in \frac{1}{\varphi(1)} \partial \varphi\left(q_{B}(\cdot)\right)\right|_{v} .
$$

Hence for any $t>0$, we have

$$
\left\langle x^{*}, t b\right\rangle=\left\langle x^{*}, v+t b\right\rangle-\left\langle x^{*}, v\right\rangle \leq \frac{1}{\varphi(1)}\left[\varphi\left(q_{B}(v+t b)\right)-\varphi\left(q_{B}(v)\right)\right] .
$$


Thus

$$
\left\langle x^{*}, b\right\rangle \leq \frac{1}{\varphi(1)} \cdot \frac{\varphi\left(q_{B}(v+t b)\right)-\varphi\left(q_{B}(v)\right)}{t} .
$$

If $q_{B}(v)=0$, then $v=0$ since $B$ is bounded. Thus (21) becomes

$$
\begin{aligned}
\left\langle x^{*}, b\right\rangle & \leq \frac{1}{\varphi(1)} \cdot \frac{\varphi\left(q_{B}(t b)\right)-\varphi\left(q_{B}(0)\right)}{t} \\
& =\frac{1}{\varphi(1)} \cdot \frac{\varphi\left(q_{B}(t b)\right)}{t}=\frac{1}{\varphi(1)} \cdot \frac{\varphi\left(q_{B}(t b)\right)}{q_{B}(t b)} \cdot q_{B}(b) .
\end{aligned}
$$

If $t \rightarrow 0^{+}$, then

$$
\frac{\varphi\left(q_{B}(t b)\right)}{q_{B}(t b)} \rightarrow \varphi^{\prime}(0)=0
$$

Thus we have

$$
\left\langle x^{*}, b\right\rangle \leq \frac{1}{\varphi(1)} \varphi^{\prime}(0) q_{B}(b)=0,
$$

which contradicts the assumption that $\left\langle x^{*}, b\right\rangle>0$. Hence we conclude that $q_{B}(v)>0$. For any $y \in B$, if there exists $t_{1}>0$ such that $q_{B}\left(v+t_{1} y\right)=q_{B}(v)$, then

$$
\left\langle x^{*}, y\right\rangle=\frac{\left\langle x^{*}, t_{1} y+v\right\rangle-\left\langle x^{*}, v\right\rangle}{t_{1}} \leq \frac{1}{\varphi(1)} \cdot \frac{\varphi\left(q_{B}\left(v+t_{1} y\right)\right)-\varphi\left(q_{B}(v)\right)}{t_{1}}=0 .
$$

If not, we have

$$
\begin{aligned}
\left\langle x^{*}, y\right\rangle & \leq \frac{1}{\varphi(1)} \cdot \frac{\varphi\left(q_{B}(v+t y)\right)-\varphi\left(q_{B}(v)\right)}{t} \\
& \leq \frac{1}{\varphi(1)} \cdot \frac{\varphi\left(q_{B}(v+t y)\right)-\varphi\left(q_{B}(v)\right)}{q_{B}(v+t y)-q_{B}(v)} \cdot q_{B}(y) \\
& \leq \frac{1}{\varphi(1)} \cdot \frac{\varphi\left(q_{B}(v+t y)\right)-\varphi\left(q_{B}(v)\right)}{q_{B}(v+t y)-q_{B}(v)} \\
& \rightarrow \frac{1}{\varphi(1)} \varphi^{\prime}\left(q_{B}(v)\right) \quad \text { as } t \rightarrow 0^{+}
\end{aligned}
$$

In any case, we have

$$
\left\langle x^{*}, y\right\rangle \leq \frac{1}{\varphi(1)} \varphi^{\prime}\left(q_{B}(v)\right), \quad \forall y \in B .
$$

On the other hand, for $0<t<1$, we have

$$
\begin{aligned}
\left\langle x^{*},-v\right\rangle & \leq \frac{1}{\varphi(1)} \cdot \frac{\varphi\left(q_{B}(v-t v)\right)-\varphi\left(q_{B}(v)\right)}{t} \\
& =\frac{1}{\varphi(1)} \cdot \frac{\varphi\left(q_{B}(v-t v)\right)-\varphi\left(q_{B}(v)\right)}{q_{B}(v-t v)-q_{B}(v)} \cdot\left(-q_{B}(v)\right) .
\end{aligned}
$$


Letting $t \rightarrow 0^{+}$, we obtain

$$
\left\langle x^{*}, v\right\rangle \geq \frac{\varphi^{\prime}\left(q_{B}(v)\right)}{\varphi(1)} \cdot q_{B}(v) .
$$

Put $u=v / q_{B}(v)$. Then $u \in B$ and

$$
\left\langle x^{*}, u\right\rangle \geq \frac{\varphi^{\prime}\left(q_{B}(v)\right)}{\varphi(1)} .
$$

Combining the above with (22), we conclude that $\left\langle x^{*}, u\right\rangle=\sup \left\langle x^{*}, B\right\rangle$.

THEOREM 4.2. Let $X$ be a locally convex space and $B \subset X$ be a weakly compact convex set with $0 \in B$. Then for any lower semicontinuous convex function $g: X \rightarrow(-\infty, \infty]$ bounded below, any $x_{0} \in \operatorname{dom} g$, any $\varepsilon>$ $g\left(x_{0}\right)-\inf g(X)$, any $\varphi \in \Phi_{2}$ and any $\lambda>0$, there exists $v \in X$ such that $q_{B}\left(v-x_{0}\right) \leq \lambda$ and

$$
g(x)+\frac{\varepsilon}{\varphi(\lambda)} \varphi\left(q_{B}\left(x-x_{0}\right)\right) \geq g(v)+\frac{\varepsilon}{\varphi(\lambda)} \varphi\left(q_{B}\left(v-x_{0}\right)\right) \quad \text { for all } x \in X .
$$

Proof. It is easy to verify that $x \mapsto g(x)+\frac{\varepsilon}{\varphi(\lambda)} \varphi\left(q_{B}\left(x-x_{0}\right)\right)$ is a lower semicontinuous convex function. Since $x_{0}+\lambda B$ is weakly compact, there exists $v \in x_{0}+\lambda B$ such that

$$
g(x)+\frac{\varepsilon}{\varphi(\lambda)} \varphi\left(q_{B}\left(x-x_{0}\right)\right) \geq g(v)+\frac{\varepsilon}{\varphi(\lambda)} \varphi\left(q_{B}\left(v-x_{0}\right)\right), \quad \forall x \in x_{0}+\lambda B .
$$

When $x \notin x_{0}+\lambda B$, we have $q_{B}\left(x-x_{0}\right)>\lambda$. For those $x$, we have $g(x)+\frac{\varepsilon}{\varphi(\lambda)} \varphi\left(q_{B}\left(x-x_{0}\right)\right)>\inf g(E)+\varepsilon>g\left(x_{0}\right) \geq g(v)+\frac{\varepsilon}{\varphi(\lambda)} \varphi\left(q_{B}\left(v-x_{0}\right)\right)$.

Thus we see that there exists $v \in X$ such that $q_{B}\left(v-x_{0}\right) \leq \lambda$ and for every $x \in X$,

$$
g(x)+\frac{\varepsilon}{\varphi(\lambda)} \varphi\left(q_{B}\left(x-x_{0}\right)\right) \geq g(v)+\frac{\varepsilon}{\varphi(\lambda)} \varphi\left(q_{B}\left(v-x_{0}\right)\right) .
$$

For convenience, if a bounded closed convex set $B$ with $0 \in B$ satisfies the hypothesis in Theorem 4.1, i.e. the perturbation $\theta(x)$ in the Borwein-Preiss principle with respect to $q_{B}$ only consists of a single summand, then $B$ is said to have the simplified Borwein-Preiss principle property (briefly, the s.b.p. property). The notion can be extended to general bounded closed convex sets. A bounded closed convex set $B$ is said to have the s.b.p. property if for some $b \in B, B-b$ has the s.b.p. property.

For a locally convex space $X$, we denote by $\left(X, \tau\left(X, X^{*}\right)\right)$ the associated Mackey space ([5, pp. 260-262]). By the James theorem (see [2, p. 77] or [4]), for any $\tau\left(X, X^{*}\right)$-complete, bounded closed convex set $B$, every $x^{*} \in X^{*}$ attains its supremum on $B$ if and only if $B$ is weakly compact. Thus in a quasi-complete locally convex space (i.e., one where each 
bounded closed set is complete, see for example [5, p. 210]), a bounded closed convex set has the s.b.p. property if and only if it is weakly compact.

This prompts us to recall another property of bounded closed convex sets, namely the drop property. Since Rolewicz [17] began the study of the drop property for closed unit balls of Banach spaces, various drop properties have been introduced and studied. For example, Giles and Kutzarova [3] defined a bounded closed convex set $B$ to have the weak drop property if for every weakly sequentially closed set $A$ disjoint from $B$ there exists a point $x_{0} \in A$ such that $D\left(x_{0}, B\right) \cap A=\left\{x_{0}\right\}$, where $D\left(x_{0}, B\right)$ denotes the convex hull of $\left\{x_{0}\right\} \cup B$. In [12], we defined a bounded closed convex set $B$ to have the quasi-weak drop property if for any weakly closed set $A$ disjoint from $B$ there exists $x_{0} \in A$ such that $D\left(x_{0}, B\right) \cap A=\left\{x_{0}\right\}$. Later we investigated the relationship between the quasi-weak drop property and weak compactness in the framework of locally convex spaces (for details, see [13]). Now, combining Theorem 4.1, Theorem 4.2 and [13, Theorem 3.2] we have the following.

THEOREM 4.3. Let $X$ be a quasi-complete locally convex space (or, let $\left(X, \tau\left(X, X^{*}\right)\right)$ be quasi-complete), and $B \subset X$ be a bounded closed convex set. Then the following statements concerning $B$ are equivalent:

(i) $B$ is weakly compact.

(ii) For any $x^{*} \in X^{*}$, there exists $u \in B$ such that $\left\langle x^{*}, u\right\rangle=\sup \left\langle x^{*}, B\right\rangle$.

(iii) $B$ has the s.b.p. property.

(iv) $B$ has the quasi-weak drop property.

From Theorem 4.3 we can deduce the following equivalent descriptions of semireflexivity and reflexivity of locally convex spaces (concerning semireflexive, reflexive and quasi-barrelled spaces, we refer to [5, §23]).

THEOREM 4.4. Let $X$ be a quasi-complete locally convex space. Then the following statements are equivalent:

(i) $X$ is semireflexive.

(ii) Every bounded closed convex set in $X$ has the quasi-weak drop property.

(iii) Every bounded closed convex set in $X$ has the s.b.p. property.

THEOREM 4.5. Let $X$ be a quasi-complete quasi-barrelled space. Then the following statements are equivalent:

(i) $X$ is reflexive.

(ii) Every bounded closed convex set in $X$ has the quasi-weak drop property.

(iii) Every bounded closed convex set in $X$ has the s.b.p. property. 


\section{References}

[1] J. M. Borwein and D. Preiss, A smooth variational principle with applications to subdifferentiability and to differentiability of convex functions, Trans. Amer. Math. Soc. 303 (1987), 517-527.

[2] K. Floret, Weakly Compact Sets, Springer, Berlin, 1980.

[3] J. R. Giles and D. N. Kutzarova, Characterisation of drop and weak drop properties for closed bounded convex sets, Bull. Austral. Math. Soc. 43 (1991), 377-385.

[4] R. C. James, Weakly compact sets, Trans. Amer. Math. Soc. 113 (1964), 129-140.

[5] G. Köthe, Topological Vector Spaces I, Springer, Berlin, 1983.

[6] M. Landsberg, Lineare topologische Räume die nicht lokalkonvex sind, Math. Z. 65 (1956), 104-112.

[7] D. Pallaschke and S. Rolewicz, Foundations of Mathematical Optimization, Math. Appl. 388, Kluwer, Dordrecht, 1997.

[8] P. Pérez Carreras and J. Bonet, Barrelled Locally Convex Spaces, North-Holland, Amsterdam, 1987.

[9] R. R. Phelps, Convex Functions, Monotone Operators and Differentiability, Springer, Berlin, 1989.

[10] J. H. Qiu, Local completeness and dual local quasi-completeness, Proc. Amer. Math. Soc. 129 (2001), 1419-1425.

[11] —, Local completeness and drop theorem, J. Math. Anal. Appl. 266 (2002), 288-297.

[12] —, On the quasi-weak drop property, Studia Math. 151 (2002), 187-194.

[13] -, On weak drop property and quasi-weak drop property, ibid. 156 (2003), 189-202.

[14] —, Ekeland's variational principle in locally complete spaces, Math. Nachr. 257 (2003), 55-58.

[15] S. Rolewicz, On a certain class of linear metric spaces, Bull. Acad. Polon. Sci. 5 (1957), 471-473.

[16] - Metric Linear Spaces, 2nd ed. revised and extended, D. Reidel and PWN-Polish Sci. Publ., Dordrecht-Warszawa, 1985.

[17] —, On drop property, Studia Math. 85 (1987), 27-35.

[18] E. Zeidler, Nonlinear Functional Analysis and its Applications III, Springer, New York, 1985.

Department of Mathematics

Suzhou University

Suzhou, Jiangsu 215006

People's Republic of China

E-mail: qjhsd@sina.com.cn
Institute of Mathematics Polish Academy of Sciences P.O. Box 21, Śniadeckich 8 00-956 Warszawa, Poland E-mail: rolewicz@impan.gov.pl

Received February 11, 2006

Revised version August 12, 2007 